Disclosure of Interests: Alexandra Damerau: None declared, Annemarie Lang: None declared, Moritz Pfeiffenberger: None declared, Timo Gaber Grant/research support from: Pfizer, Frank Buttgereit: None declared DOI: 10.1136/annrheumdis-2019-eular.5834

\section{FRI0508 OLIVE OIL POLYPHENOLS AS NOVEL NUTRACEUTICALS IN TREATMENT OF OSTEOARTHRITIS}

Mia S. Meiss ${ }^{1}$, Marina Sanchez-Hidalgo ${ }^{2}$, Catalina Alarcón-de-la-Lastra ${ }^{2}$, José M. Fernández-Bolaños ${ }^{2}$, Richard Oc Oreffo ${ }^{1}$, Maria C de Andres ${ }^{1,3} .{ }^{1}$ Centre for Human Development, Stem Cells and Regeneration, Institute of Developmental Sciences, Southampton, United Kingdom; ${ }^{2}$ University of Seville, Faculty of Pharmacy, Department of Pharmacology, Seville, Spain; ${ }^{3}$ Biomedical Research Institute of A Coruña-INIBIC, A Coruña, Spain

Background: Osteoarthritis $(O A)$ is a degenerative disease of the joints characterised by the imbalance of anabolic and catabolic processes in articular cartilage. It is one of the most significant causes of disability in the world affecting over $60 \%$ of people over the age of 60 , causing joint stiffness, pain and a decrease in the quality of life. Currently no successful disease-modifying agent has been found to prevent or treat the condition. To address this unmet need, alternative approaches, including the use of nutraceuticals as a novel therapeutic intervention are under examination.

Objectives: To analyse if nutraceutical are able to reverse the catabolic activity that contribute to cartilage destruction in OA. Here two polyphenols from extra virgin olive oil (EVOO), oleocanthal (OLC) and ligstroside aglycone (LA), plus a chemically modified acetylated ligstroside aglycone (A-LA), and two marine polyunsaturated fatty acids, eicosapentaenoic acid (EPA) and docosahexaenoic acid (DHA), were examined as potential antiinflammatory agents for $O A$.

Methods: Human chondrocytes and cartilage explants were extracted from OA femoral heads, cultured and incubated with increasing concentrations of select compounds in combination with the pro-inflammatory cytokines $\mathrm{IL}-1 \beta$ and oncostatin $M$ (OSM). Gene expression levels were analysed by qPCR; nitric oxide (NO), GAG release and cytokines were measured in supernatants by colorimetric assays and Luminex technology respectively. Safranin-O and Alcian blue/Sirius red staining were done to quantify extracellular matrix components from cartilage.

Results: Acetylated ligstroside showed the most promising results for implementation in treating $O A$ as it reduced the expression of pro-inflammatory genes such as inducible nitric oxide (iNOS), matrix metalloprotease-13 (MMP13) and interleukin-1 $\beta$ (IL1B) at both RNA and protein levels; decreased nitric oxide (NO) levels from cartilage explants and also reduced proteoglycan (PG) losses in human osteoarthritic cartilage explants and chondrocytes (Figure 1).

Conclusion: These results substantiate the role of nutraceuticals in OA with implications for therapeutic intervention and our understanding of $O A$ pathophysiology.

\section{REFERENCES :}

[1] Castrogiovanniet al. Nutraceutical supplements in the management and prevention of osteoarthritis. Int. J. Mol. Sci. 17, (2016).

[2] Rigacci \& Stefani. Nutraceutical Properties of Olive Oil Polyphenols. An Itinerary from Cultured Cells through Animal Models to Humans. Int. J. Mol. Sci. 17, 1-28 (2016).

[2] Aparicio-Soto, et al. Extra virgin olive oil: a key functional food for prevention of immune-inflammatory diseases. Food Funct. 7, 4492-4505 (2016).

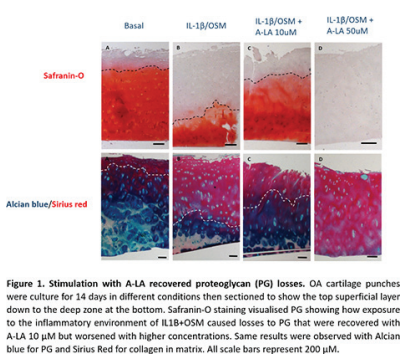

Disclosure of Interests: None declared DOI: 10.1136/annrheumdis-2019-eular.7078

\section{FRI0509 \\ 3D MICROSTRUCTURE OF INTACT AND OSTEOARTHRITIC HUMAN MENISCUS USING MICRO- COMPUTED TOMOGRAPHY}

lida Kestilä ${ }^{1}$, Elin Folkesson ${ }^{2}$, Mikko A. Finnilä ${ }^{1}$, Aleksandra Turkiewicz ${ }^{2}$, Patrik Önnerfjord ${ }^{2}$, Velocity Hughes ${ }^{2}$, Jon Tjörnstrand ${ }^{2}$, Martin Englund ${ }^{2,3}$,

Simo Saarakkala ${ }^{1,4}$. 'University of Oulu, Oulu, Finland'; ${ }^{2}$ Lund University, Lund, Sweden; ${ }^{3}$ Boston University School of Medicine, Boston, United States of America; ${ }^{4}$ Oulu University Hospital, Oulu, Finland

Background: Degenerative meniscal lesions are highly prevalent in the general population and are associated with increased risk of knee osteoarthritis (OA). Development of novel methods for visualization of the early degenerative changes in the meniscus could help us better understand meniscal degradation and the onset of OA.

Objectives: To develop and perform ex vivo 3D imaging of meniscus posterior horn microstructure with micro-computed tomography $(\mu \mathrm{CT})$, and to compare specimens from healthy references with end-stage $\mathrm{OA}$ using histology and qualitative $\mu \mathrm{CT}$.

Methods: Medial and ipsilateral lateral menisci were retrieved from 10 patients undergoing total knee arthroplasty for medial compartment knee OA (age range 50 to 75 years, 5 women). Additionally, medial menisci were obtained from 10 cadaveric donors without known $\mathrm{OA}$, to serve as reference (age range 18 to 77 years, 5 women). The samples were freshly frozen in $-80^{\circ} \mathrm{C}$. After thawing, the posterior horns were dissected and fixed, followed by excision of a piece for $\mu \mathrm{CT}$ imaging (Fig. 1A). The $\mu \mathrm{CT}$ samples were dehydrated in ascending ethanol concentrations, treated with hexamethyldisilazane (HMDS), and dried in room temperature. They were then imaged with a desktop $\mu \mathrm{CT}$ (scanning parameters: 40 $\mathrm{kV}, 250 \mu \mathrm{A}, 2.0 \mu \mathrm{m}$ pixel size, $1815 \mathrm{~ms}$, no additional filtering). For histological analysis, Pauli's histopathological scoring ${ }^{1}$ was performed independently by two graders on sections from three regions (Fig. 1A). The graders' consensus was given for each sub-score, from which the final overall consensus scores were calculated for each region. The mean difference in consensus scores $(95 \% \mathrm{Cl})$, adjusted for age and sex, were estimated between the menisci from $\mathrm{OA}$ knees and the reference menisc (medial OA menisci vs reference menisci, and lateral menisci from OA patients vs reference menisci) using a mixed linear regression model. The mean difference in consensus scores between the medial and lateral menisci from the same OA patient was calculated using a linear fixed effects model. The models were fitted on all three histological regions, but here we report histology results from region 2, because this region is adjacent to the piece analyzed by $\mu \mathrm{CT}$ (Fig 1A).

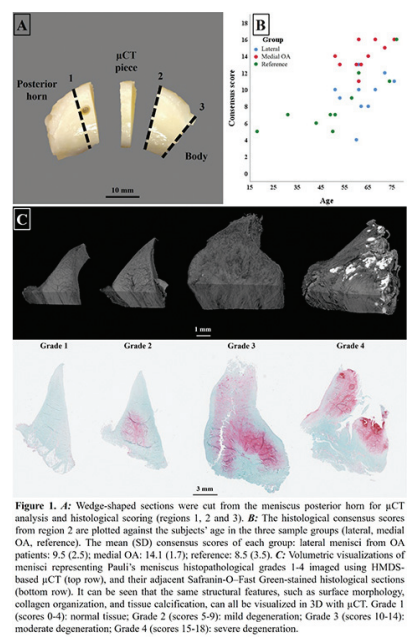

Results: 3D visualization of the meniscus with $\mu \mathrm{CT}$ revealed similar struc tural changes as histological analysis (Fig. 1C). The histopathological consensus scores were generally higher in medial OA samples as compared to both lateral menisci from OA patients and the reference menisci (Fig. 1B). The mean difference in histological scores $(95 \% \mathrm{Cl})$ was $4.3(2.5$, 6.1) for medial $O A$ menisci vs reference menisci, $-0.3(-2.2,1.5)$ for lateral menisci from $\mathrm{OA}$ patients vs reference menisci, and $4.6(3.0,6.2)$ for medial OA menisci vs lateral menisci from OA patients.

Conclusion: An HMDS-based 3D $\mu \mathrm{CT}$ protocol allows unique 3D visualization of meniscus microstructures. The method enables the visualization of considerably large volumes and evaluation of the tissue's 3D 
organization. Therefore, it may provide additional, important information compared to conventional section-based histology. Moreover, analyzing patients with medial compartment knee OA, we found that the medial OA menisci had higher histopathological scores than both reference medial menisci, as well as the lateral meniscus from the same knee.

\section{REFERENCES:}

[1] Pauli C, Grogan SP, Patil S, Otsuki S, Hasegawa A, Koziol J, et al. Macroscopic and histopathologic analysis of human knee menisci in aging and osteoarthritis. Osteoarthritis Cartilage 2011;19:1132-41. Doi: 10.1016/j. joca.2011.05.008 [doi]

Acknowledgement: We would like to acknowledge the Foundation for Research in Rheumatology (FOREUM) for funding.

Disclosure of Interests: None declared

DOI: 10.1136/annrheumdis-2019-eular.4110

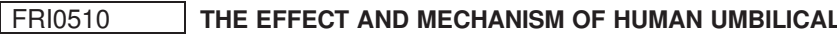 CORD MESENCHYMAL STEM CELLS-DERIVED EXOSOMES ON BONE DESTRUCTION OF COLLAGEN INDUCED ARTHRITIS RATS}

Jinfang Gao, Ke Xu, Gailian Zhang, Jian Han, Yang Liu, Liyun Zhang. Shanxi Dayi Hospital, Rheumatology, Taiyuan, China

Background: Rheumatoid arthritis (RA) is a highly disabling autoimmune disease, characterized by destruction of the cartilage and bone, which is difficult to reverse. mesenchymal stem cells-derived exosomes (Mesenchymal stem cells-derived Exos) is a kind of extracellular vesicle, produced by MSCs in resting or stress state, which can simulate the tissue repair effect of its maternal cells and may be a new breakthrough point in the treatment of RA bone destruction.

Objectives: Study the effect of human umbilical cord mesenchymal stem cells derived exosomes(hUCMSC-exos) on bone destruction in Collagen induced arthritis (CIA) rats.

Methods: 1. After isolated by differential centrifugation, hUCMSCs was cultured in vitro, which was identified by morphology and surface markers.2. Grouping: The CIA rats were randomly divided into CIA group, MTX group, hUCMSCs group, hUCMSC-exos low concentration group, hUCMSC-exos medium concentration group, hUCMSC-exos high concentration group, and a healthy control group.3. Efficacy evaluation: the efficacy of hUCMSC-exos on CIA rats was evaluated by measuring joint swelling, arthritis index, micro-ct scanning and pathological score. The expression levels of RANKL and OPG in the serum of rats in each group were detected by high-throughput flow,multi-factor detection technique or ELISA method, and the levels of the above factors in the synovial tissue of the ankle were detected by immunohistochemical detection technique, and the mRNA levels were detected by RT-PCR.

Results: 1. Effect evaluation: hUCMSC-exos can improve the arthritis index $(\mathrm{Al})$ of $\mathrm{ClA}$ rats, and no definite side effect been find. In addition to hUCMSC-exos low concentration group, the joint synovial hyperplasia and inflammatory cell infiltration degree of each treatment group were significantly improved, comparing with that of CIA group. The Results of Micro-ct scanning suggested that the degree of osteoporosis in each intervention group was significantly improved comparing with that in the CIA group, and the improvement degree of hUCMSCs groups,MTX groups and hUCMSC-exos high concentration group was similar.2.The detection of bone metabolism factor in Serum and synovial tissue: medium and high concentrations of hUCMSC-exos, hUCMSCs group and MTX group could down-regulate the level of RANKL in serum and synovial tissues of CIA rats, and raise the level of OPG, and the effect of hUCMSC-exos in high concentration group was slightly obvious.

Conclusion: hUCMSC-exos can improve bone destruction in CIA rats, and it is safe. hUCMSC-exos can mimic its mother cells and exert an inhibitory effect on bone destruction by regulating the imbalance of RANKL/ OPG, and the effect is equal or better than its mother cells, and the high concentration group is superior to the low concentration group.

\section{REFERENCES:}

[1] Aghajani NA, Lerman LO, Eirin A. Mesenchymal stem cell-derived extracellular vesicles for kidney repair: current status and looming challenges. STEM CELL RES THER 2017;8(1):273.

[2] Calabresi E, Petrelli F, Bonifacio AF, Puxeddu I, Alunno A. One year in review 2018: pathogenesis of rheumatoid arthritis. CLIN EXP RHEUMATOL 2018;36(2):175-84.

[3] Emori T, Hirose J, Ise K, Yomoda JI, Kasahara M, Shinkuma T, Yoshitomi $H$, Ito $H$, Hashimoto $M$, Sugahara S, Fujita $H$, Yamamoto $N$, Morita $Y$,
Narumiya S, Aramori I. Constitutive Activation of Integrin alpha9 Augments Self-Directed Hyperplastic and Proinflammatory Properties of Fibroblastlike Synoviocytes of Rheumatoid Arthritis. J IMMUNOL 2017;199(10):3427 36.

[4] Potter DR, Miyazawa BY, Gibb SL, Deng X, Togaratti PP, Croze RH, Srivastava AK, Trivedi A, Matthay M, Holcomb JB, Schreiber MA, Pati S Mesenchymal stem cell-derived extracellular vesicles attenuate pulmonary vascular permeability and lung injury induced by hemorrhagic shock and trauma. J Trauma Acute Care Surg 2018;84(2):245-56.

Disclosure of Interests: None declared

DOI: 10.1136/annrheumdis-2019-eular.7325

FRI0511

LOW $\Omega 6$ - $\Omega 3$ RATIOS CAUSED REDUCTION IN CELLULAR INFLAMMATION IN HUMAN SYNOVIOCYTES CELL LINE (SW982): GENE EXPRESSION STUDY

Abhay Harsulkar ${ }^{1}$, Priya Kulkarni ${ }^{2}$, Vanshika Srivastava ${ }^{3}$, Ragini Vidya ${ }^{3}$, Saurabh Yadav ${ }^{3}$, Shreya Chitnavis ${ }^{3}$, Alankruta Senapati ${ }^{3} .{ }^{1}$ Bharati Vidyapeeth University, Department of Cell and Molecular Biology, Pune, India; ${ }^{2}$ University of Tartu, Department of Pathophysiology, Tartu, Estonia; ${ }^{3}$ Bharati Vidyapeeth University, Department of Pharmaceutical Biotechnology, Pune, India

Background: Alarming global increase in osteoarthritis $(O A)$, coupled with off target existing palliative care poses nutraceuticals an extremely attrac tive alternative in this respect. Beyond meeting the basic nutritiona demands, nutrition is thought to play a beneficial role in the managemen of chronic diseases. Interestingly, the off target effects of nutriceuticals are beneficial in reducing co-morbidities. Skewed ratio of $\omega-6: \omega-3$ fatty acids (FAs) in modern diets has been shown associated with increased number of inflammatory diseases including bone and cartilage metabolism. Considering this, due to their disease modifying actions, $\omega-3$ FAs are thought to serve better in OA management.

Objectives: We here investigated the effect of different $\omega-6$ : $\omega-3$ ratios on synovitis, a prominent OA pathology. The selected ratios are generally found in global diet

Methods: Selected $\omega-6$ FAs - arachidonic acid (AA), eicosadienoic acid (EDA), linolic acid (LA) and dihomo gamma linoleic acid (DGLA) along with selected $\omega$-3 FAs docosapentaenoic acid 5 (DPA) and $\alpha$-linolenic acid (ALA) were tested on induced SW-982 cells, in common dietary ratios of $16: 1,8: 1,4: 1,2: 1$ and $1: 1$ for their effect on synovial inflammation. For induction, $50 \mathrm{ng} / \mathrm{ml}$ tumor necrosis factor- $\alpha$ (TNF- $\alpha$ ) was used for 72 hrs and the PUFAs were added in the culture media in the selected

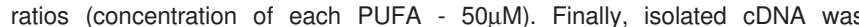
used to run quantitative RT-PCR for superoxide dismutase (SOD), interleukin-15 (IL-15), matrix metalloproteinase-1 (MMP-1) and vascular endothelial growth factor (VEGF).

Results: All the ratios LA:ALA of caused a remarkable reduction in MMP $1(\mathrm{P}<0.001)$. Marginal reduction in VEGF was noted with $1: 1,4: 1$ and $8: 1$ ratios $(P<0.005) ; 8: 1$ and $16: 1$ showed a significant increase $(P<0.001)$ SOD did not show any particular trend but noteworthy increase was observed with $2: 1,8: 1$ and $16: 1$ ratios $(P<0.001)$ while reduction with $1: 1$ and $4: 1$ ratios $(P<0.001)$. IL-15 remained low for $1: 1,2: 1$ and $4: 1$ while a marked up-regulation was noted for $8: 1$ and $16: 1$ ratios $(P<0.001)$. In case of EDA:DPA, a consistent down-regulation of MMP-1was found with all the ratios excluding $4: 1$. The selected ratios were efficient against VEGF $(P<0.001)$. A consistent high SOD was revealed by all the ratios of EDA:DPA, which remained comparable to control. Marginal reduction in IL-15 was noted with $1: 1, \quad 8: 1$ and $16: 1 \quad(P<0.05)$. Lastly, a significant MMP-1 reduction was caused by all the ratios of DGLA:DPA, except 2:1 Excluding 2:1, all the ratios were efficient against VEGF $(P<0.001)$ Increased levels of SOD remained comparable with TNF- $\alpha$ for $1: 1$ and $4: 1$; a trivial drop was noted with $2: 1$ and $8: 1$, followed by a noticeable reduction with $16: 1$. IL-15 showed a down-regulation with $1: 1,4: 1,8: 1$ ratios of DGLA: DPA.

Conclusion: Consequence of synovial inflammation in typical and atypical forms results in cartilage-loss, osteophytes and pain. FA ratios when used in equivalent, was found highly effective against IL 15, MMP1 and VEGF, whereas inflammation increases with increase in their proportions Low SOD indicated lower oxidative-stress while it was up-regulated in response to high stress. FAs work in two distinct ways, membrane incorporation and metabolic modulation. Unsaturated fatty acid increase membrane fluidity and thus improves cell signaling. Analysis of treated and untreated cells showed incorporation of FAs in cell membrane, which improves membrane fluidity and status of signal transduction.

Acknowledgement: This research work has been carried out as a part of OAIBGE2016-18, an international consortium supported by INNO- 